\title{
CONTROLE POPULACIONAL DE CÃES E GATOS EM DEZ VILAS RURAIS DO PARANÁ: RESULTADOS EM MÉDIO PRAZO
}

\section{(Populational control of dogs and cats in ten Rural Villages in the State of Paraná, Brazil: mid-term results)}

\author{
MOLENTO, C. F. M¹.; LAGO, E. ${ }^{2}$; BOND, G.B. ${ }^{2}$ \\ 1 Departamento de Zootecnia (LABEA), UFPR, carlamolento@yahoo.com. \\ 2 Mestrandos em Ciências Veterinárias, UFPR.
}

\begin{abstract}
RESUMO - Devido à necessidade de maior entendimento sobre a duração de esforços para o controle da população de animais de companhia, formulou-se um questionário com o objetivo de verificar o impacto em médio prazo de um projeto de esterilização cirúrgica (PEC), realizado para auxiliar no controle das populações de cães e gatos em dez Vilas Rurais (VRs) na região noroeste do estado do Paraná. O questionário foi aplicado para toda a população das VRs trabalhadas, em média três anos e três meses após o PEC. Adicionalmente, em uma VR foi pesquisado o número de habitantes por moradia, com o objetivo de se calcular os índices cão:ser humano e gato:ser humano. Os índices cão:ser humano e gato:ser humano encontrados foram de 1:2,7 e 1:5,2, respectivamente. Os resultados mostram que os esforços para o controle populacional de cães e gatos devem ser avaliados em médio e longo prazo para que todos os fatores envolvidos sejam compreendidos. Apenas $21 \%$ da população esterilizada durante o PEC ainda estavam presentes; desta forma, um limitante importante para o controle populacional de cães e gatos é o fluxo destes animais nas VRs. Para que futuras intervenções apresentem efeitos duradouros é essencial que se fomente o aumento da expectativa de vida dos animais esterilizados e que se controle a chegada de novos animais, visando a diminuição do fluxo de cães e gatos nas VRs. Em conclusão, os resultados são coerentes com a perspectiva de que a solução para o descontrole populacional de cães e gatos reside na educação para guarda responsável.
\end{abstract}

Palavras-chave: bem-estar; cão; companhia; gato; população.

\begin{abstract}
Due to the need for more information regarding the duration of efforts towards pet population control, a questionnaire was developed to verify the mid-term impact of a surgical sterilization project (SSP), conducted to help dog and cat population control in ten Rural Villages (RVs) in the Northeastern region of the State of Paraná, Brazil. The questionnaire was applied to all participants RV's inhabitants, on average three years and three months after the SSP. Additionally, in one of the RVs, the number of residents per house was asked, with the objective of calculating the dog: human being and cat: human being ratios. Results show that the efforts towards pet population control must be evaluated in mid and long-term if all the factors involved are to be understood. Only $21 \%$ of the sterilized population during the SSP were still present; thus, an important limiting factor for pet population control is the flow of dogs and cats in the RVs. The dog: human being and cat: human being ratios were 1:2.7 and 1:5.2, respectively. In order to promote longer lasting effects for future interventions, it is essential to foster strategies to increase life expectation of sterilized animals and to control the arrival of new animals, with an overall goal of decreasing pet flow in the RVs. Results agree with the perspective that the key factor for pet population control is the education towards responsible pet guardianship.
\end{abstract}

Key-words: cat; companion; dog; pet; population; welfare.

1 E-mail: carlamolento@yahoo.com

R. dos Funcionários, 1540 CEP 80035-050 - Juvevê, Curitiba, PR Fone (41) 3350-5788 - FAX (41) 3350-5629 


\section{INTRODUÇÃO}

O descontrole da população de cães e gatos representa um problema nos centros urbanos, comprometendo o bem-estar humano e animal. Cães e gatos nas ruas trazem riscos de zoonoses, agressão e danos à propriedade (CARDING, 1969; CARTER, 1990; CIAMPI e GARCIA, 1996); adicionalmente, os animais estão sujeitos a acidentes de trânsito, fome, frio, abusos e maus tratos. Tais problemas tornam essencial um controle populacional efetivo. O método mais difundido de controle populacional foi, por muito tempo, a captura e extermínio. Animais recolhidos por centros de controle de zoonoses eram exterminados por não serem considerados passíveis de adoção ou por excederem o contingente de adoções da população (MOLENTO et al., 2005).

Recentemente, a Organização Mundial da Saúde emitiu relatório sobre os métodos de controle populacional de cães, no qual declara ineficiente a captura e extermínio (WHO, 2005). A partir desta constatação e de preocupações com o bem-estar dos cães de rua, emerge a necessidade de pesquisa para a construção de alternativas mais eficazes e humanitárias para o controle populacional de cães em ambientes urbanos. Assim, novas estratégias de controle populacional de cães de rua estão sendo expostas nos meios acadêmicos e profissionais da área. Algumas propostas envolvem esterilização cirúrgica, educação pública para guarda responsável e aplicação de legislação pertinente. Em particular, em cenários nos quais a guarda responsável de animais de companhia depende de estratégias de médio e longo prazo, faz-se necessário o desenvolvimento de métodos de controle populacional que incorporem a presença de cães nas ruas, como por exemplo a vasectomia (MOLENTO, 2004). Idealmente, as estratégias a serem propostas devem fundamentar-se no conhecimento de dinâmica populacional e de comportamento animal, assim como no reconhecimento dos animais como seres sencientes.

No Brasil, a Carta São José dos Pinhais, redigida pelos participantes do VII Curso de Formação de Oficiais de Controle Animal e Publicada pelo Conselho Regional de Medicina Veterinária do Paraná (CRMV, 2006), reconhece que é necessária uma mudança nas estratégias para o controle populacional de animais no Brasil e que cães de rua esterilizados, vacinados e desverminados oferecem à comunidade uma barreira reprodutiva e sanitária. Os animais aceitos pela comunidade podem ser recolhidos, esterilizados, vacinados, identificados, desverminados e devolvidos ao local em que estavam, desde que não estejam em risco ou coloquem em risco outros animais, seres humanos e meio ambiente e tenham, se possível, alguém da comunidade ou instituições que assumam a responsabilidade pelos mesmos.

Programas de esterilização em massa vem sendo realizados, no sentido de reduzir a população de cães e gatos semi-domiciliados aptos à reprodução. Entretanto, existe uma necessidade de maior entendimento sobre a duração de esforços para o controle da população de animais de companhia. A evidência sugere baixo impacto em médio prazo, uma vez que as populações de cães apresentam uma expectativa de vida média a baixa, de dois anos e seis meses em Baltimore, EUA (BECK, 2002) e de dois anos e quatro meses em Araucária, PR (WOLFF, 2005). Outros fatores que contribuem para a ineficácia destes programas em médio prazo são a alta taxa de reposição da população e o número insuficiente de animais esterilizados.

O objetivo deste trabalho foi verificar o impacto de um projeto de esterilização cirúrgica (PEC) (MOLENTO et al., 2005) após um período médio de três anos e três meses, visando uma contribuição para a construção de programas de controle populacional de cães e gatos com maiores chances de sucesso em médio e longo prazo.

\section{MATERIAL E MÉTODOS}

Formulou-se um questionário constituído de 14 perguntas (QUADRO 1) com o objetivo de estudar a população atual de cães e gatos nas dez VRs participantes do PEC. O questionário englobou três perguntas sobre a participação dos proprietários no PEC, oito perguntas sobre a situação atual dos animais nas residências e da população de cães e gatos das VRs, enfocando ainda a guarda responsável, e três perguntas sobre a opinião dos proprietários com relação à importância do PEC na VR. As entrevistas foram realizadas durante o mês de julho de 2005, sendo que os moradores das VRs foram entrevistados em suas casas. Moradores de todas as casas das VRs participantes do PEC, após em média três anos e três meses da primeira intervenção, foram entrevistados. Os dados obtidos foram estudados através de estatística descritiva, utilizando-se o programa Microsoft Office Excel 2003. 
Controle populacional de cães e gatos em dez Vilas Rurais do Paraná:

resultados em médio prazo

QUADRO 1 -QUESTIONÁRIO APLICADO EM DEZ VILAS RURAIS (VRS) DO NOROESTE DO PARANÁ, APÓS TRÊS ANOS E TRÊS MESES DE UM PROJETO DE ESTERILIZAÇÃO CIRÚRGICA DE CÃES E GATOS (PEC), EM JULHO DE 2005.

Acompanhamento após três anos e três meses do PEC, realizado de 2001 a 2003

Questionário

Vila Rural:

Data:

1

Morador: Casa/lote $n^{\circ}$ :

Entrevistador:

01. Você participou do projeto de controle populacional de cães e gatos (PEC)?

02. Participou de alguma reunião ou palestra sobre o assunto?

03. Quantos animais você tem atualmente? (separar cães e gatos, machos e fêmeas)

04. Há quanto tempo cada um dos animais está com você?

05. Algum animal seu foi inscrito no projeto? (separar cães e gatos, machos e fêmeas)

06. Algum animal inscrito não está mais com você? Por qual motivo?

07. Como está seu animal, teve alguma doença?

08. Houve aquisição de novos animais depois que o projeto atuou? Quantos?

09. Houve nascimentos de cães ou gatos em sua casa? Ou em algum lugar da vila?

10. Havia mais nascimentos antes do projeto? Sim ( ) Não ( ) Não sabe ( )

11. Apareceram cães ou gatos abandonados na vila? Quantos?

12. A cirurgia dos animais trouxe algum beneficio para a sua vila? Qual?

13. Você considera necessária a continuação desse projeto?

14. O que você achou do projeto?

Na VR José Francisco de Oliveira foi adicionada uma pergunta ao questionário para pesquisar o número de habitantes por moradia, com o objetivo de se calcular os índices cão:ser humano e gato:ser humano.

\section{RESULTADOS E DISCUSSÃO}

As entrevistas nas VRs, realizadas no período de 18/07/2005 a 22/07/2005, seguiram a ordem cronológica apresentada na (TABELA 1). O menor intervalo entre o PEC e as entrevistas foi na VR Pedro Urkia, de dois anos e quatro meses, e o maior foi na VR Nossa Terra, de quatro anos e dois meses.

Os resultados obtidos revelaram uma população total de cães de 414 sendo 172 (41,5\%) fêmeas e $242(58,5 \%)$ machos. O total de gatos foi de 154 sendo $57(37,1 \%)$ fêmeas e 97 (62,9\%) machos. As populações de cães e gatos detalhadas por VR encontram-se na TABELA 2. Comparando-se os dados atuais com aqueles do PEC, verificou-se que a população total aumentou de 518 para 568 cães e gatos nas dez VRs e que $116(35,2 \%)$ moradores adotaram novos animais.

Os dados encontrados mostram que existe um alto índice de êxodo de famílias das VRs, pois apenas os moradores de $101(68,7 \%)$ das $147 \mathrm{ca}$ sas que participaram do PEC estavam presentes. Desta forma, deve-se levar em consideração um fluxo de famílias relativamente alto nas VRs, fator provavelmente associado ao alto fluxo de animais. Uma vez que o êxodo familiar representa um fator importante na diminuição do número de animais esterilizados nas VRs, é provável que estes tenham colaborado com o controle de nascimentos de cães e gatos em outras localidades, das quais não se tem informações. Ainda, o número de famílias que não participaram do PEC aumentou após três anos e três meses, devido à chegada de novos moradores e do êxodo de alguns dos que participaram na época do PEC (TABELA 3). 
TABELA 1 - VISITAS DO ACOMPANHAMENTO DO PROJETO DE ESTERILIZAÇÃO CIRÚRGICA (PEC), REALIZADO EM DEZ VILAS RURAIS (VRS) NO NOROESTE DO PARANÁ, NO PERÍODO DE 18/07/2005 A 22/07/2005, COM INTERVALO MÉDIO DE TRÊS ANOS E TRÊS MESES.

\begin{tabular}{ccc}
\hline Vila Rural & Localidade & $\begin{array}{c}\text { Intervalo entre o PEC e as } \\
\text { entrevistas }\end{array}$ \\
\hline Pedro Urkia & Santa Elisa & 2 anos e 3 meses \\
T. Fugiharu & Serra dos Dourados & 2 anos e 9 meses \\
August Herwig & Herculândia & 2 anos e 10 meses \\
Nova Jerusalém & Perobal & 3 anos \\
Ricardo Brunelli & Maria Helena & 3 anos \\
Primavera & Cafezal do Sul & 3 anos e 1 mês \\
Primavera & Icaraíma & 3 anos e 9 meses \\
São Carlos & Lovat & 3 anos e 11 meses \\
José F. de Oliveira & Xambrê & 3 anos e 11 meses \\
Nossa Terra & Cianorte & 4 anos e 2 meses \\
\hline
\end{tabular}

TABELA 2 - POPULAÇÃO DE CÃES E GATOS, EM DEZ VILAS RURAIS (VRS) NA REGIÃO NOROESTE DO PARANÁ, APÓS INTERVALO MÉDIO DE TRÊS ANOS E TRES MESES DE UM PROJETO DE ESTERILIZAÇÃO CIRÚRGICA DE CÃES E GATOS (PEC), EM JULHO DE 2005.

\begin{tabular}{ccccc}
\hline Vila Rural - Município & Cães & Cadelas & Gatos & Gatas \\
\hline Nossa Terra - Cianorte & 24 & 9 & 3 & 0 \\
São Carlos - Lovat & 24 & 12 & 3 & 1 \\
J. F. de Oliveira - Xambrê & 27 & 28 & 17 & 9 \\
Primavera - Icaraíma & 13 & 6 & 3 & 1 \\
Primavera - Cafezal do Sul & 20 & 3 & 5 & 1 \\
Ricardo Brunelli - Maria Helena & 29 & 32 & 9 & 7 \\
Nova Jerusálem - Perobal & 22 & 14 & 10 & 6 \\
August H. Herwig - Herculândia & 19 & 19 & 8 & 13 \\
T. Fugiharu - Serra dos Dourados & 32 & 19 & 12 & 14 \\
Pedro Urkia - Santa Elisa & 32 & 30 & 27 & 5
\end{tabular}


Controle populacional de cães e gatos em dez Vilas Rurais do Paraná:

resultados em médio prazo

TABELA 3 - SITUAÇÃO DAS MORADIAS NAS DEZ VILAS RURAIS (VRS) DO NOROESTE DO PARANÁ, APÓS UM PERÍODO MÉDIO DE TRÊS ANOS E TRÊS MESES DE UM PROJETO DE ESTERILIZAÇÃO CIRÚRGICA DE CÃES E GATOS (PEC), EM JULHO DE 2005.

\begin{tabular}{cccccccccccc}
\hline Moradias & $\begin{array}{c}\text { Nossa } \\
\text { Terra }\end{array}$ & $\begin{array}{c}\text { São } \\
\text { Carlos }\end{array}$ & $\begin{array}{c}\text { J. F. de } \\
\text { Oliveira }\end{array}$ & $\begin{array}{c}\text { Primavera } \\
\text { (Icaraima) }\end{array}$ & $\begin{array}{c}\text { Primavera } \\
\text { (Cafezal do } \\
\text { Sul) }\end{array}$ & $\begin{array}{c}\text { Ricardo } \\
\text { Brunelli }\end{array}$ & $\begin{array}{c}\text { Nova } \\
\text { Jerusalém }\end{array}$ & $\begin{array}{c}\text { A. H. H. } \\
\text { Herwig }\end{array}$ & $\begin{array}{c}\text { Tokogiru } \\
\text { Fugiharu }\end{array}$ & $\begin{array}{c}\text { Pedro } \\
\text { Urkia }\end{array}$ & TOTAL \\
\hline $\begin{array}{c}\text { Com participação } \\
\text { no PEC }\end{array}$ & 8 & 7 & 10 & 4 & 7 & 14 & 8 & 10 & 16 & 18 & $\mathbf{1 0 2}$ \\
$\begin{array}{c}\text { Sem participação } \\
\text { no PEC }\end{array}$ & 9 & 16 & 33 & 10 & 11 & 24 & 20 & 18 & 22 & 10 & $\mathbf{1 7 9}$ \\
$\begin{array}{c}\text { Sem animais } \\
\text { Número total de }\end{array}$ & 1 & 2 & 4 & 4 & 4 & 12 & 8 & 2 & 7 & 10 & $\mathbf{5 4}$ \\
\hline moradias & 18 & 25 & 47 & 18 & 22 & 50 & 36 & 30 & 45 & 38 & $\mathbf{3 2 9}$ \\
\hline
\end{tabular}

Dentre os animais esterilizados durante o PEC, somente $21 \%$ estavam presentes, sendo $21(8,0 \%)$ cães; $16(6,1 \%)$ cadelas; $11(4,2 \%)$ gatos e $7(2,7 \%)$ gatas. De um total de 261 animais esterilizados, $31,3 \%$ estavam ausentes com motivo conhecido e $47,7 \%$ estavam ausentes sem motivo conhecido, totalizando $79 \%$ de animais ausentes após um período de três anos e três meses do PEC. A maioria dos proprietários não soube justificar a ausência de seus animais (FIGURA 1), sugerindo que os conceitos de guarda responsável encontram-se pouco fundamentados na população das VRs.

Os motivos declarados para ausência dos animais esterilizados foram morte por envenenamento em 9,6 $\%$ dos animais, morte por causa desconhecida em $5,0 \%$, doação em 3,8\%, atropelamento em 3,4\% e outras causas em $9,5 \%$ (TABELA 4 ).

\section{FIGURA 1 - PRESENÇA DOS ANIMAIS ESTERILIZADOS APÓS INTERVALO MÉDIO DE TRÊS ANOS E TRÊS MESES DE UM PROJETO DE ESTERILIZAÇÃO CIRÚRGICA DE CÃES E GATOS (PEC), EM DEZ VILAS RURAIS (VRS) NO NOROESTE DO PARANÁ, EM JULHO DE 2005.}

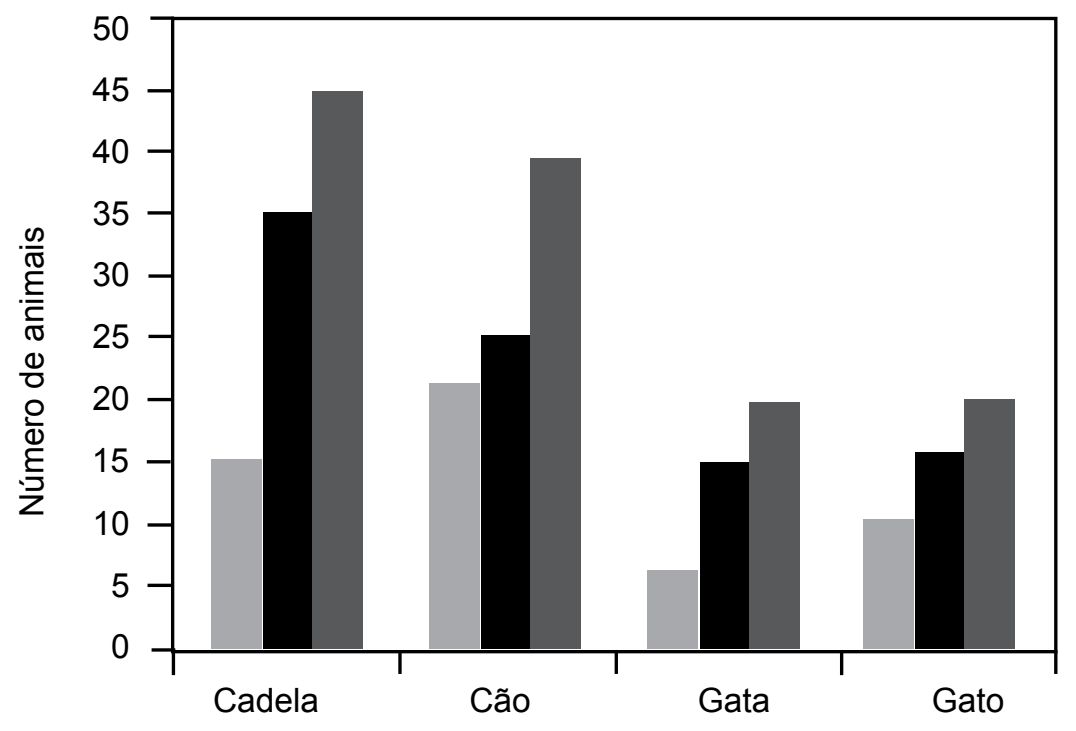

Animais presentes

- Animais ausentes com informação

- Animais ausentes sem informações 
TABELA 4 - MOTIVO DAAUSÊNCIA DOS ANIMAIS DE ACORDO COM O RELATO DOS PROPRIETÁRIOS EM DEZ VILAS RURAIS (VRS) DO NOROESTE DO PARANÁ APÓS TRÊS ANOS E TRÊS MESES DE UM PROJETO DE ESTERILIZAÇÃO CIRÚRGICA DE CÃES E GATOS (PEC), EM JULHO DE 2005.

\begin{tabular}{cccc}
\hline $\begin{array}{c}\text { Motivo da Ausência } \\
\text { do animal }\end{array}$ & $\begin{array}{c}\text { Número de } \\
\text { animais }\end{array}$ & $\begin{array}{c}\text { Em relação ao total de } \\
\text { animais ausentes com } \\
\text { informação (\%) }\end{array}$ & $\begin{array}{c}\text { Em relação ao total de 261 animais } \\
\text { esterilizados no PEC (\%) }\end{array}$ \\
\hline Envenenamento & 25 & 30,5 & 9,6 \\
Doação & 13 & 15,8 & 5,0 \\
Atropelamento & 11 & 13,4 & 4,2 \\
Doente & 6 & 7,4 & 2,3 \\
Velhos & 6 & 7,4 & 2,3 \\
Desaparecidos & 5 & 6,1 & 1,9 \\
Mortos & 5 & 6,1 & 1,9 \\
Cirurgia & 4 & 4,9 & 1,5 \\
Virose & 3 & 3,6 & 1,1 \\
Roubo & 2 & 2,4 & 0,8 \\
Tumor & 2 & 2,4 & 0,8 \\
\hline Total & 82 & 100 & 31,4 \\
\hline
\end{tabular}

O alto percentual de ausência dos animais esterilizados durante o PEC e o aumento numérico da população de cães e gatos são indicativos de uma alta taxa de reposição de cães e gatos nas VRs, associada a uma provável baixa expectativa de vida dos animais. Outro fator que colabora com o aumento no fluxo de animais, de acordo com o relato dos moradores, é a alta taxa de cães e gatos provenientes de outras regiões que são abandonados nas VRs.

O número médio de habitantes por moradia na VR José Francisco de Oliveira foi de 3,2. Com base na média de habitantes por moradia, os índices cão:ser humano e gato:ser humano foram de 1:2,7 e 1:5,2, respectivamente. Tais índices são coerentes com aqueles de outras cidades brasileiras e da América Latina, divergindo de maneira importante dos números do continente africano (TABELA 5). Desta forma, a produção de um índice médio para países em desenvolvimento parece inadequada, sendo necessária a diferenciação de países da América Latina em relação aos países africanos. A tendência nos países africanos é de um índice cão:ser humano menor que o encontrado nas Vilas Rurais, sendo este fator provavelmente devido à grande população de seres humanos nos países africanos (KNOBEL et al., 2005).
Pôde-se observar um efeito positivo para o bemestar de cães e gatos nas VRs em função do PEC, ainda que o fluxo de animais tenha sido alto e a população tenha revelado um aumento numérico após três anos e três meses. Segundo (BEAVER, 2001), uma única cadela não castrada pode dar origem direta e indiretamente a 67.000 cães por um período de seis anos. Sendo assim, com a esterilização de 93 cadelas durante PEC, teoricamente foram evitados 6.231.000 nascimentos durante um período de seis anos. Desta forma, é razoável concluir que a taxa de natalidade foi reduzida em função do número de animais esterilizados no PEC. Este raciocínio, associado à pequena alteração do número total de cães e gatos ao longo de três anos e três meses e ao alto porcentual de animais esterilizados ausentes ao final deste período, sugere que a maioria dos filhotes não sobrevive, sendo que as causas de morte na maioria das vezes são desconhecidas. Em outras palavras, a diminuição da taxa de natalidade promoveu uma diminuição da taxa de mortalidade, uma vez que a população se manteve relativamente constante ao longo de três anos e três meses. Isto sugere uma provável diminuição de sofrimento animal na situação de descontrole das populações de cães e gatos nas VRs do noroeste do Paraná devido ao PEC. 
Controle populacional de cães e gatos em dez Vilas Rurais do Paraná:

resultados em médio prazo

TABELA 5 - ÍNDICES CÃO: SER HUMANO EM DIFERENTES LOCALIDADES DO BRASIL E DO EXTERIOR.

\begin{tabular}{llcl}
\hline Estado / País & Cidade ou região & Razão Cão: Ser humano & Referência \\
\hline Paraná & Cidades do noroeste & $1: 2,7$ & MOLENTO et al.(2005) \\
Paraná & Piraquara & $1: 2,3$ & BIONDO et al. (2007) \\
Paraná & Curitiba & $1: 3,3$ & DAMASCO et al. (2005) \\
Paraná & Araucária & $1: 4$ & WOLFF (2005) \\
São Paulo & Taboão da Serra & $1: 5,1$ & DIAS et. al. (2004) \\
São Paulo & Araçatuba & $1: 3,6$ & NUNES et al. (1997) \\
São Paulo & Serra Azul & $1: 5$ & MATOS et al. (2002) \\
São Paulo & Ibiúna & $1: 3,8$ & SOTO et al. (2003) \\
Minas Gerais & Ouro Preto & $1: 2,6$ & NAVEDA et al.(2002) \\
Argentina & Almirante Brown & $1: 4,0$ & ANTONIAZZI (2005) \\
México & Baja Califórnia & $1: 4,3$ & FLORES-IBARRA e \\
Quênia & Machakos District & $1: 9,6$ & ESTRELA-VALENZUELA (2004) \\
Zâmbia & Ambiente urbano & $1: 45,0$ & DE BAL al. (1993) \\
\hline
\end{tabular}

\section{CONCLUSÕES}

Os resultados mostram que não houve impacto em médio-prazo do PEC no controle populacional de cães e gatos nas VRs, sendo que a população total aumentou de 518 para 568 cães e gatos nas dez VRs. Após três anos e três meses do PEC estavam presentes apenas $21 \%$ dos animais esterilizados. Esta baixa proporção de animais esterilizados presentes ocorreu devido à morte dos animais e ao fluxo de moradores. A proporção de animais esterilizados ausentes associada à relativa constância do número total de animais revela que a taxa de reposição da população é alta. Adicionalmente, considerando-se uma população animal relativamente constante $e$ uma menor taxa de natalidade devido ao PEC, houve provável redução de sofrimento animal devido à redução na taxa de mortalidade. Para que futuras intervenções apresentem efeitos mais duradouros, é essencial que sejam desenvolvidas estratégias para o aumento da expectativa de vida dos animais esterilizados e que se controle a chegada de novos animais, visando a diminuição do fluxo de cães e gatos nas VRs. Os resultados são coerentes com a perspectiva de que a solução para o descontrole populacional de cães e gatos reside na educação para guarda responsável.

\section{REFERÊNCIAS}

ANTONIAZZI, M. Controle populacional de cães e gatos - uma experiência internacional bem sucedida. Palestra proferida pela Presidente de la Asociación Amigos Del Centro Municipal de Sanidad Animal Y Zoonosis, durante o $1^{\circ}$. Seminário Nacional Novas Diretrizes para os Centros de Controle de Zoonoses - Animais e Saúde Pública, Câmara Municipal de Belo Horizonte, 2005.

BEAVER, B.V. Comportamento Canino - Um Guia para Veterinários. São Paulo: Roca, 2001, 431 p.

Beck, A. The ecology of stray dogs: a study of freeranging urban animals. West Lafayette: Purdue University Press, 2002, 98 p.

BIONDO, A.W.; CHARELLO, T.; LARSEN, H.; GOMIG, T.; UCHIDA, L.; LOSSO, M.; BARROS, A. C. R.; PIMENTEL, J. S.; JAVOROUSKI, E. B.; RIBEIRO, K.G.; BRANCO, I. D.; WOUK, A. F. P. F. Censo canino em Piraquara, Paraná. Livro de resumos do $15^{\circ}$. EVINCI, UFPR, 2007 (no prelo). 
CARDING, A.H. The significance and dynamics of stray dog populations with special reference to the U.K. and Japan. Journal Small Animal Practice, v. 10, p. 419-446, 1969.

CARTER, C.N. Pet population control: another decade without solutions? Journal of the American Veterinary Medical Association, v.197, p. 192-195, 1990.

CIAMPI, M.A.S.; GARCIA, R.C.M. Campanha de controle das populações de cães e gatos no município de Taboão da Serra, São Paulo, Brasil. Arca Brasil - Associação Humanitária de Proteção e Bem-Estar Animal e Prefeitura de Taboão da Serra, Relatório técnico 1996.

CRMV PR. Conselho Regional de Medicina Veterinária do Estado do Paraná. Carta de São José dos Pinhais. Curitiba. n²1, ano V. p.27, 2006.

DAMASCO, R.T.; BIONDO, A.W.; KOBLITZ, E.; FAGUNDES, C.L.; STAUDACHER, C.; PLAHINSCE, C.R.S.; VALEIXO, M.; MOLENTO, C.F.M. Controle populacional de cães na Vila Osternack, município de Curitiba, PR. Livro de resumos do $13^{\circ}$. EVINCI, UFPR, p. 66, 2005.

DE BALOGH, K.K.; WANDELER, A.I.; MESLIN, F.X. A dog ecology study in an urban and a semirural area of Zambia. Onderstepoort Journal of Veterinary Research and Animal Science. v.4, n. 60, p. 437-443, 1993.

DIAS, R.A.; GARCIA, R.C.; SILVA, D.F.; AMAKU, M.; FERREIRA-NETO, J.S.; FERREIRA, F. Estimativa de populações canina e felina domiciliadas em zona urbana do Estado de São Paulo. Revista Saúde Pública. v. 4, n. 38, p. 565-570, 2004.

FLORES-IBARRA, M.; ESTRELLA-VALENZUELA, G. CANINE Ecology and socioeconomic factors associated with dogs unvaccinated against rabies in a Mexican City across the US-Mexico border. Preventive Veterinary Medicine. v.2, n. 62, p.79-87, 2004.

KITALA, P.M.; MCDERMOTT, J.J.; KYULE, M.N.; CATHUMA, J.M. Features of dog ecology relevant to rabies spread in Machakos District, Kenya. Onderstepoort Journal of Veterinary Research and Animal Science. v. 4, n. 60, p.445-449, 1993.
KNOBEL, D. L.; CLEAVELAND, S.; COLEMAN, P.G.; FĖVRE, E.M.; MELTZER, M.I.; MIRANDA, M.E.G.; SHAW, A.; ZINSSTAG, J.; MESLIN, F.X. Re-evaluating the burden of rabies in Africa an Asia. Bulletin of the Word Health Organization, 2005.

MATOS, M.R.; ALVES, M.C.G.P.; REICHMANN, M.L.A.B.; DOMINGUEZ, M.H.S. Técnica Pasteur São Paulo para dimensionamento de população canina. Caderno de Saúde Pública. v. 5 , n. 18, p. 1423-1428, 2002.

MOLENTO, C.F.M. Vasectomising stray dogs. Veterinary Record, v.155 n. 20 p. 648, 2004.

MOLENTO, C.F.M.; INOE A.P.; REGO, M.I.C.; LAGO, E.; MEZA S.K.L.; LEME, M.C.; MOLENTO, M.B. Controle populacional de cães e gatos em dez Vilas Rurais do Paraná, Brasil. Arquivo de Ciências Veterinárias e Zoologia da Unipar, v. 8, $n^{\circ} .1, p$. 25-31, 2005.

NAVEDA, L.A.B.; MOREIRA, E.C.; VIANA, F.C.; SILVA, J.A.; PEREIRA, P.L. Avaliação do risco de Leishmaniose visceral nas comunidades de Glaura e Soares, Ouro Preto, MG, 2002. Escola de Veterinária da UFMG, 2002.

NUNES, C.M.; MARTINES, D.A.; FIKARIS, S.; QUEIROZ, L.H. Evaluation of the dog population in na urban area of southeastern Brazil. Revista Saúde Pública. v. 3, n. 31, p. 308-309, 1997.

SOTO, F.R.M. Dinâmica populacional canina no Município de Ibiúna-SP: estudo restropectivo de 1998 a 2002 referente a animais recolhidos, eutanasiados e adotados, 2003.

WHO. World Health Organization Expert Consultation on Rabies. WHO Technical Report Series, 931, First Report. 2005. Genebra.

WOLFF, F. Comunicação pessoal (2005).

Recebido para publicação:

$30 / 08 / 2007$ Aprovado:

$05 / 12 / 2007$ 